\title{
STABILITY OF GIRDER BRIDGE AGAINST TSUNAMI FLUID FORCE
}

\author{
Susumu Araki ${ }^{1}$, Kazuo Ishino² and Ichiro Deguchi ${ }^{1}$
}

\begin{abstract}
The horizontal and vertical components of the fluid force and pressure acting on a girder bridge due to tsunami like a solitary wave are measured in a hydraulic experiment and their characteristics are discussed. The peak of the impact fluid force is estimated on the basis of the change in the added mass of the structure. The wave pressure acting on the seaward side of the girder of the bridge is also estimated. The critical force for the stability of the girder bridge is estimated as the strength of bolts used in the shoes against shear stress.
\end{abstract}

Keywords: bridge; tsunami; fluid force; wave pressure; impact force

\section{INTRODUCTION}

In Japan, several tsunamis have caused severe damage to coastal zone. It has been pointed out that large scale earthquakes will occur near Japan and tsunamis caused by the earthquakes will strike coasts in the near future. In many coastal zones in Japan, mountains are very close to the sea and main roads have often been constructed along the coast lines. If a bridge across a river is washed away due to a tsunami, it is difficult to set up a diversion in several areas because of mountainous topography. Therefore, the stability of bridges and the countermeasures to prevent bridges from being washed away have to be investigated. In the present design of bridge beams, tsunami fluid force has not been taken into account. The characteristics of tsunami fluid force acting on bridge beams are different from that acting on coastal and harbor structures, such as breakwaters and seawalls because there is clearance between the structures and the still water level.

Many studies on tsunami fluid forces acting on coastal and harbor structures have been conducted. Cross (1967) investigated the impact forces acting on vertical walls. Ramsden and Raichlen (1990) measured the fluid force and pressure due to bore. Matsutomi (1991) also measured the wave pressure due to bore acting on vertical walls. Asakura et al. (2000) measured the fluid force due to overflowing tsunami acting on structures constructed behind seawalls. Mizutani and Imamura (2000) measured the wave pressure due to bore acting on sloping structures. Ikeno et al. (2005) measured the wave pressure due to tsunami with soliton fission acting on vertical breakwaters. Mizutani et al. (2006) measured the tsunami fluid force acting on containers on aprons in a harbor. Nouri et al. (2010) measured the fluid force and pressure due to bore acting on both cylindrical and square structures placed on a dry bed. On the contrary, few studies on tsunami fluid force acting on bridge beams which have clearance under them have been conducted (Shoji and Mori, 2006; Araki et al., 2008; Araki and Deguchi, 2009).

The aim of this study is to measure the tsunami fluid force acting on girder bridges which are widely adapted in relatively small rivers and to investigate the stability of girder bridges against tsunami fluid force.

\section{EXPERIMENTAL SET-UP}

Hydraulic experiments were conducted in a two-dimensional wave flume which is $41.0 \mathrm{~m}$ long and $0.7 \mathrm{~m}$ wide in Osaka University. Figure 1 shows a rough sketch of the experimental set-up. The bottom slopes of the sea and the river were $1 / 40$ and $1 / 100$, respectively. The incident wave like a solitary wave was generated by piston type wave generator. The tsunami height $a$ was defined as the maximum rise in the water surface as shown in Figure 2. The directions of the horizontal and the vertical fluid forces $F_{x}$ and $F_{z}$ are also illustrated in Figure 2. In this experiment, the tsunami height in front of the model girder bridge $a$ was ranged from $7.8 \mathrm{~cm}$ to $14.2 \mathrm{~cm}$. Table 1 shows the still water depth at the model girder bridge $h$ and the clearance under the bridge $h_{c}$ used in the experiments.

Figure 3 illustrates the model girder bridge. The model bridge has three main girders. Figure 4 is a photograph of the reverse side of the model girder bridge. The reverse side of the model bridge is like a grid. The horizontal and vertical components of the tsunami fluid force acting on the model bridge were measured by force transducer. Figure 5 is a photograph of the model bridge installed in the wave flume. The wave pressure on the model bridge was also measured at four points from P1 to P4 shown

\footnotetext{
${ }^{1}$ Department of Civil Engineering, Osaka University, 2-1 Yamada-oka, Suita, Osaka, 565-0871, Japan

2 Taisei Technology Center, Taisei Corporation, 344-1 Nase-cho, Totsuka, Yokohama, Kanagawa, 245-0051, Japan
} 
in Figure 3 by pressure gauges. However, the measurements of the tsunami fluid force by force transducer and the wave pressure by pressure gauge could not be conducted simultaneously for the reason of the measuring instrument. The water surface displacement was measured by capacitance-type wave gauge. The distance from the model bridge to the wave gauge just in front of the bridge was 0.10 $\mathrm{m}$. The measured data were digitized at the time interval of $0.002 \mathrm{~s}(500 \mathrm{~Hz})$ and were recorded by a data acquisition system.

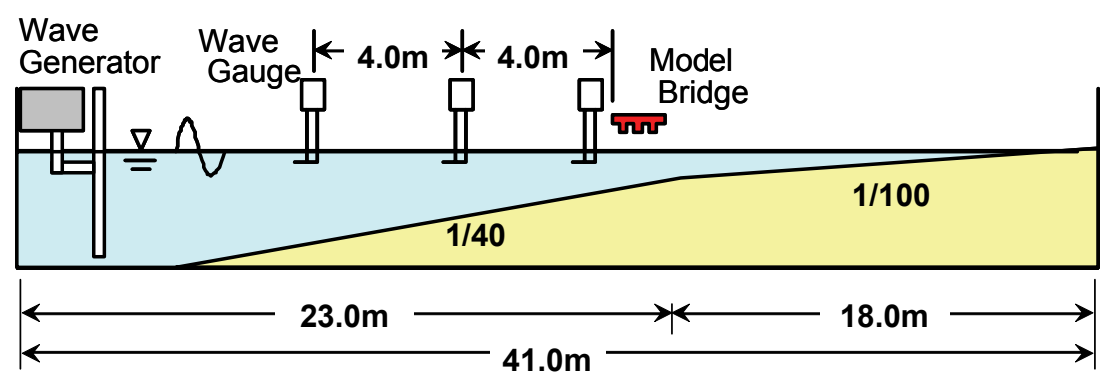

Figure 1. Experimental Set-up.

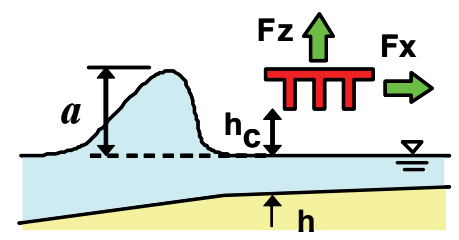

Table 1. Water depth at bridge $h$ and clearance hc.

\begin{tabular}{|c|c|}
\hline $\mathrm{h}(\mathrm{cm})$ & $\mathrm{h}_{\mathrm{c}}(\mathrm{cm})$ \\
\hline 16.0 & 13.5 \\
\hline 18.0 & 11.5 \\
\hline 20.0 & 9.5 \\
\hline 22.0 & 7.5 \\
\hline 24.0 & 5.5 \\
\hline
\end{tabular}

Figure 2. Definition sketch of parameters.

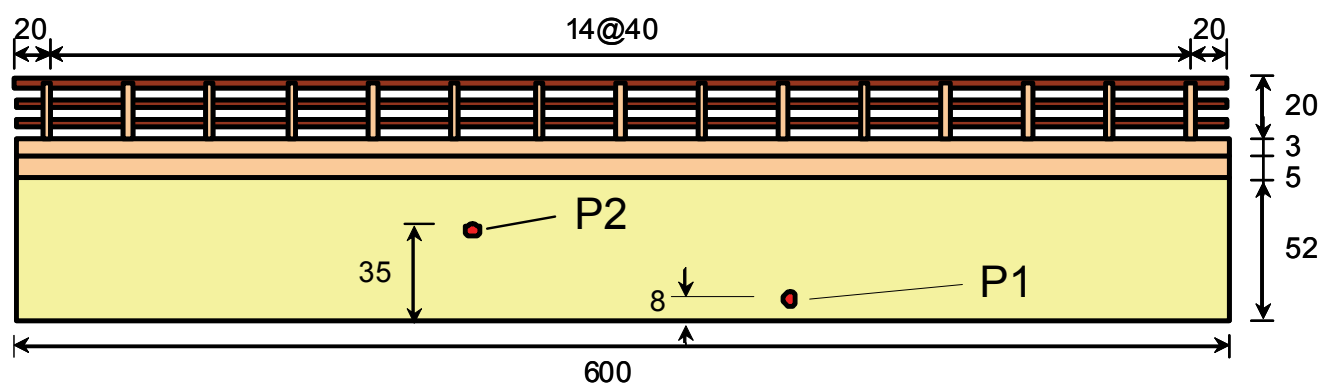

(a) Front view.

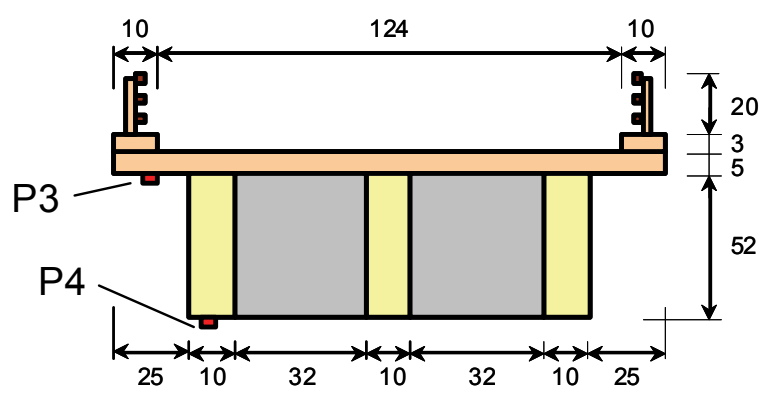

(b) Cross-section.

Figure 3. Model girder bridge (unit; $\mathbf{m m}$ ). 


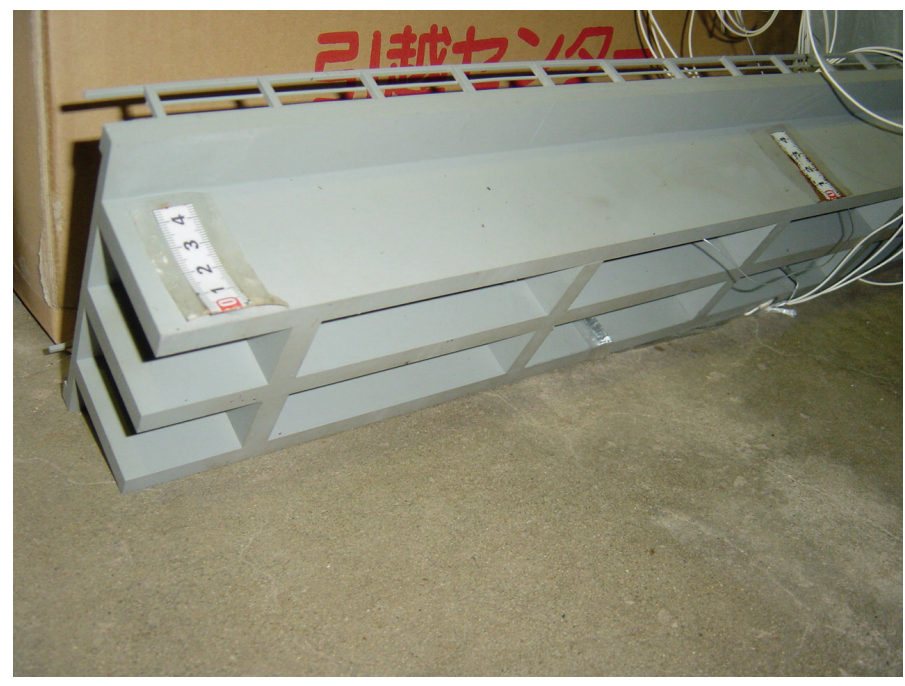

Figure 4. Reverse side of the model girder bridge.

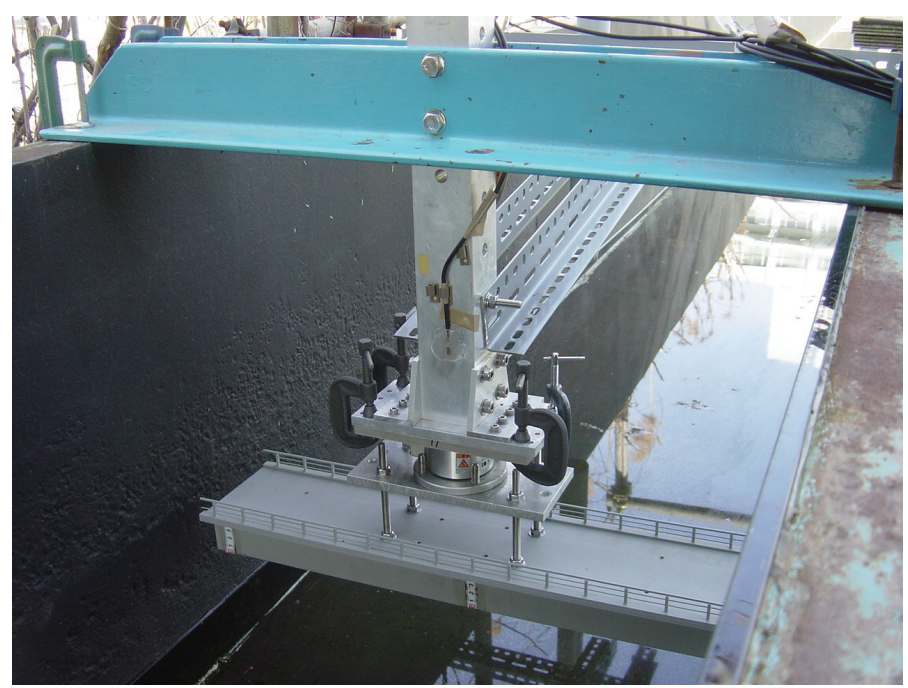

Figure 5. Model girder bridge installed in the flume.

\section{MEASURED FLUID FORCE AND WAVE PRESURRE}

\section{Time histories}

Figure 6 shows typical examples of time histories of the vertical components of the fluid force acting on the model bridge $F_{z}$ and the water surface displacement in front of the model bridge $\eta$. In the figure, the red and blue lines show the vertical fluid force and the water surface displacement, respectively. In Figure 6(a), a just breaking wave struck the model bridge while a post breaking wave struck the model bridge in Figure 6(b). The tsunami heights in Figures 6(a) and 6(b) were $a=12.6 \mathrm{~cm}$ and $14.4 \mathrm{~cm}$, respectively. The depth at the model bridge was $h=18.0 \mathrm{~cm}$ and the clearance between the model bridge and the still water level was $h_{c}=11.5 \mathrm{~cm}$ in both cases. The impact force was measured at the moment when the water surface struck the model bridge. The magnitude of the impact force is very large; however, the duration is very short. In the time history of the vertical fluid force $F_{z}$ 
shown in Figure 6(a), a downward force was measured after an upward impact force. In the case of a just breaking wave shown in Figure 6(a), two peaks were measured in the time history of the vertical fluid force. On the other hand, in the case of a post breaking wave shown in Figure 6(b), just one peak was measured in the time history of the vertical fluid force. In the time history of the horizontal fluid force $F_{x}$, the number of the peak of the impact was not so clear because the natural frequency of the force measuring device in the horizontal direction was not as high as that in the vertical direction.

Figure 7 shows typical examples of time histories of the wave pressure from P1 to $\mathrm{P} 4$ on the model bridge. Figures 7(a) and 7(b) show time histories of the wave pressure on the model bridge in the cases of $a=9.8 \mathrm{~cm}, h=22.0 \mathrm{~cm}, h_{c}=7.5 \mathrm{~cm}$ and $a=13.6 \mathrm{~cm}, h=22.0 \mathrm{~cm}, h_{c}=7.5 \mathrm{~cm}$, respectively. The wave pressures $\mathrm{P} 1$ and $\mathrm{P} 2$ acting on the seaward side of the main girder correspond to the horizontal fluid force. It seems that the wave pressure P3 was mainly exerted by the up-rush of the water which struck the seaward side of the main girder. The $\mathrm{P} 4$ is the wave pressure acting on the reverse side of the main girder. In the case shown in Figure 7(a), the magnitude of the peak of P4 was as large as those of P1, P2 and P3. However, the magnitude of the peak of P4 was smaller than those of P1, P2 and P3 in many cases. The magnitude of the peak of P3 was as large as those of P1 and P2 in several cases. The peak of P4 was earlier than those of P1, P2 and P3 in all the cases. However, the order of the peaks of $\mathrm{P} 1, \mathrm{P} 2$ and $\mathrm{P} 3$ depended on the test conditions.

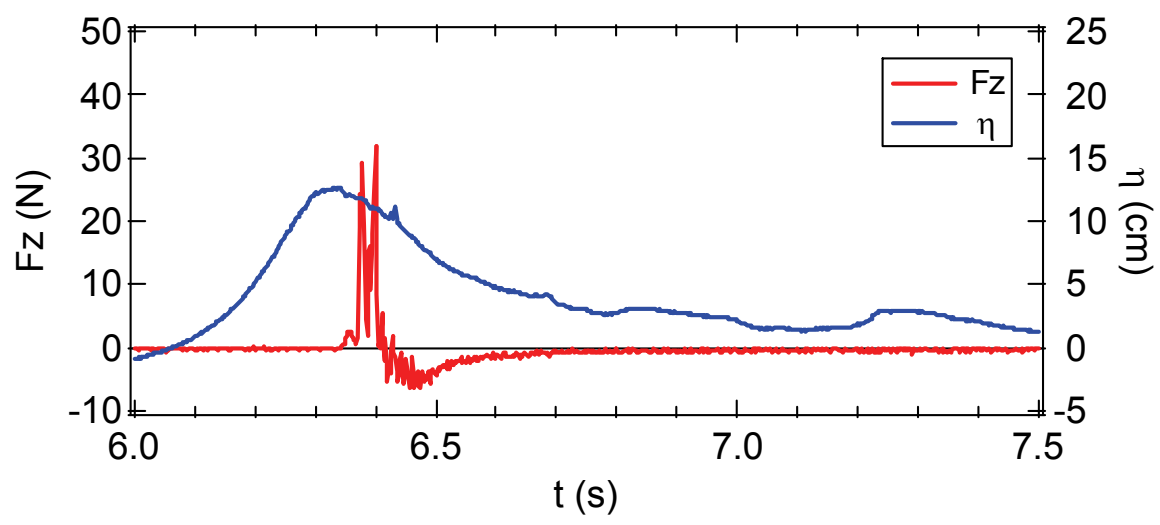

(a) $\mathrm{a}=12.6 \mathrm{~cm}, \mathrm{~h}=18.0 \mathrm{~cm}$ and $\mathrm{hc}=11.5 \mathrm{~cm}$

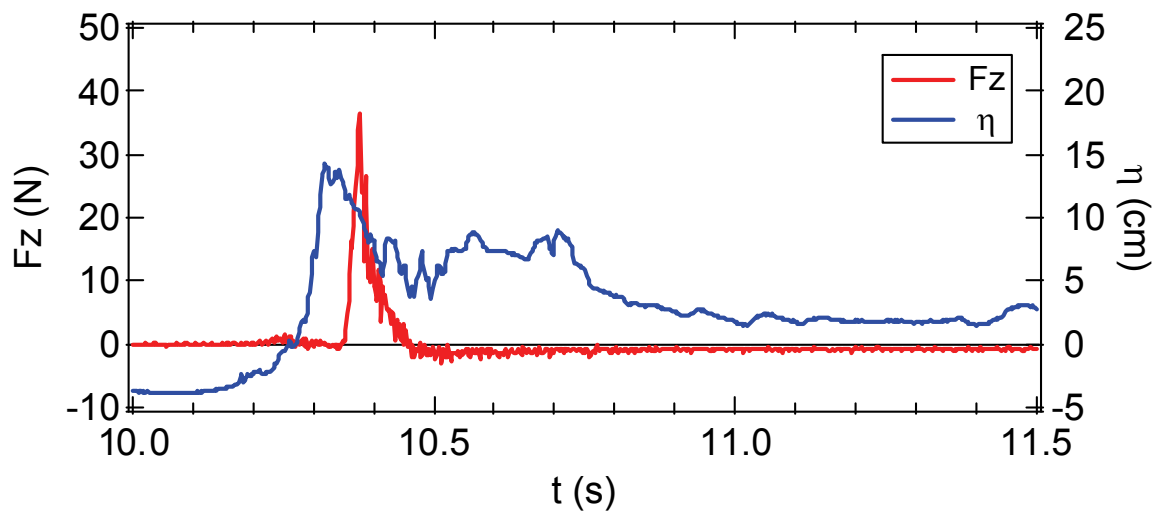

(b) $\mathrm{a}=14.4 \mathrm{~cm}, \mathrm{~h}=18.0 \mathrm{~cm}$ and $\mathrm{hc}=11.5 \mathrm{~cm}$

Figure 6. Time histories of vertical fluid force and water surface displacement in front of model bridge. 


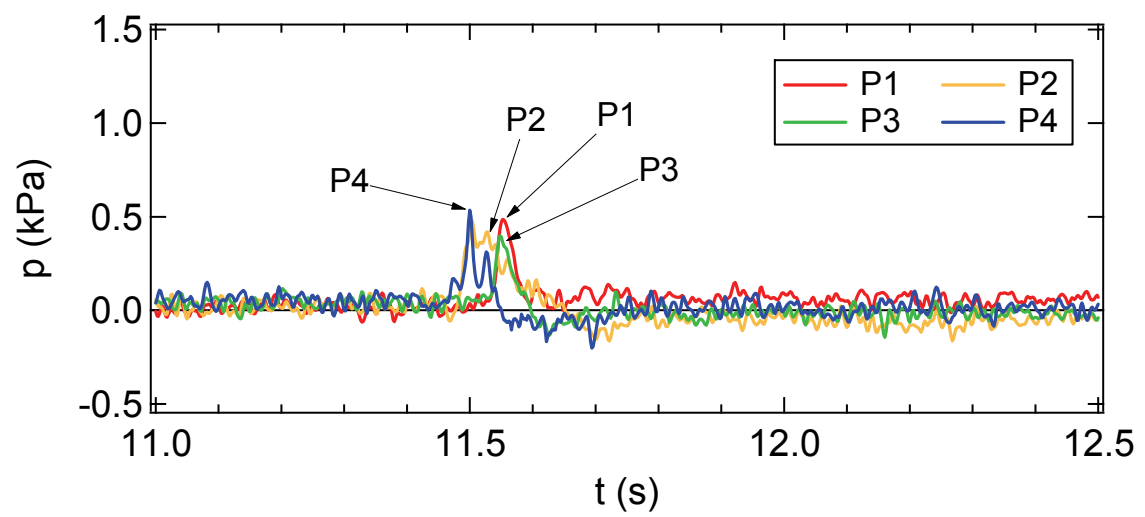

(a) $\mathrm{a}=9.8 \mathrm{~cm}, \mathrm{~h}=22.0 \mathrm{~cm}$ and $\mathrm{hc}=7.5 \mathrm{~cm}$

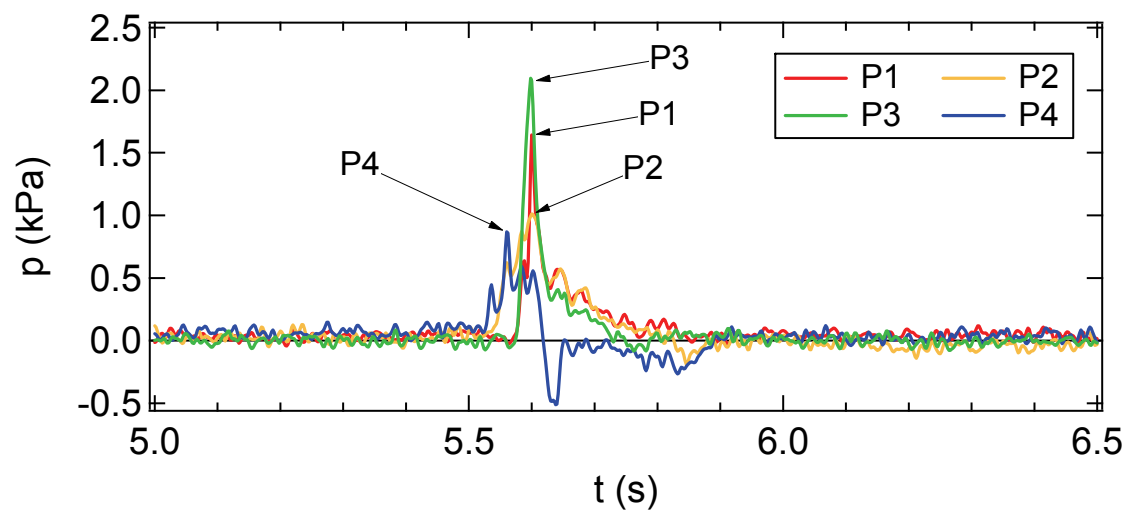

(b) $\mathrm{a}=13.6 \mathrm{~cm}, \mathrm{~h}=22.0 \mathrm{~cm}$ and $\mathrm{hc}=7.5 \mathrm{~cm}$

Figure 7. Time histories of wave pressure on model bridge.

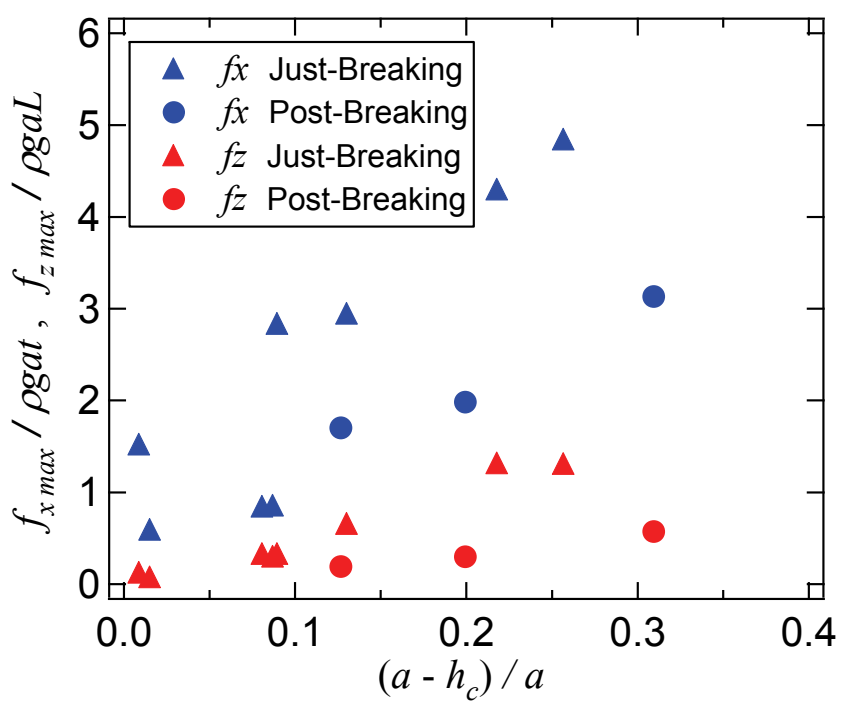

Figure 8. Relationship between maximum fluid forces and tsunami height above model bridge. 


\section{Characteristics of Fluid Force}

Figure 8 shows a relationship between the maximum measured force and the tsunami height above the model bridge. The horizontal axis shows the tsunami height above the model bridge normalized by the tsunami height $a$. The vertical axis shows the maximums of the horizontal and vertical components of the measured forces acting on the model bridge per unit length $f_{x \max }$ and $f_{z \max }$ normalized by the water density $\rho$, the gravitational acceleration $g$, the tsunami height $a$ and the height of the girder of the model bridge $t$ for $f_{x}$ or the width of the model bridge $L$ for $f_{z}$. The blue symbol shows the maximum of the horizontal measured force and the red symbol shows the maximum of the vertical measured force. The triangle shows the maximum force measured in the case of a just breaking wave and the circle shows the maximum force measured in the case of a post breaking wave. In both horizontal and vertical directions, the maximum force in the case of a post breaking wave was smaller than that in the case of a just breaking wave.

\section{Estimation of Impact Force}

Impulsive forces exerted by the impact of water surface on structures are generally estimated on the basis of the change in the added mass of the structures in the transition from emerged to submerged conditions. When an object falls into the still water at the constant falling velocity $v$, the fluid force including an impact force acting on the object $F$ is estimated by the following equation.

$$
F=\frac{d}{d t}\left(m_{a} \cdot v\right)=m_{a} \frac{d v}{d t}+v \frac{d m_{a}}{d t}
$$

where $m_{a}$ is the added mass of the object.

In a completely submerged object, the fluid force is expressed by only the first term because the added mass of the object does not change with time. However, the added mass of the object changes with time just after the impact of the water surface on the object. In other words, the second term corresponds to the impact force. In this study, the added mass of the model bridge was calculated as half of the infinitely long cylinder shown in Figure 9. The angle of the water surface to the still water level was calculated from the time history of the water surface displacement measured in front of the model bridge. The velocity $v$ in Eq. (1) was calculated from the time history of the water surface displacement and the angle of the water surface to the still water level because it corresponded to the rising velocity of the water surface.

Figure 10 shows the comparison between the peaks of the measured and the estimated impact fluid forces. The red circle shows the impact force for just breaking waves and the blue circle shows the impact force for post breaking waves. Figure 10(a) shows the comparison in the horizontal component. Although the impact forces for post breaking waves were estimated on the safe side in most cases, the impact forces for just breaking waves were considerably underestimated in several cases. Figure 10(b) shows the comparison in the vertical component. The trend in the vertical component is similar to that in the horizontal component. However, the degree of the scattering is smaller.

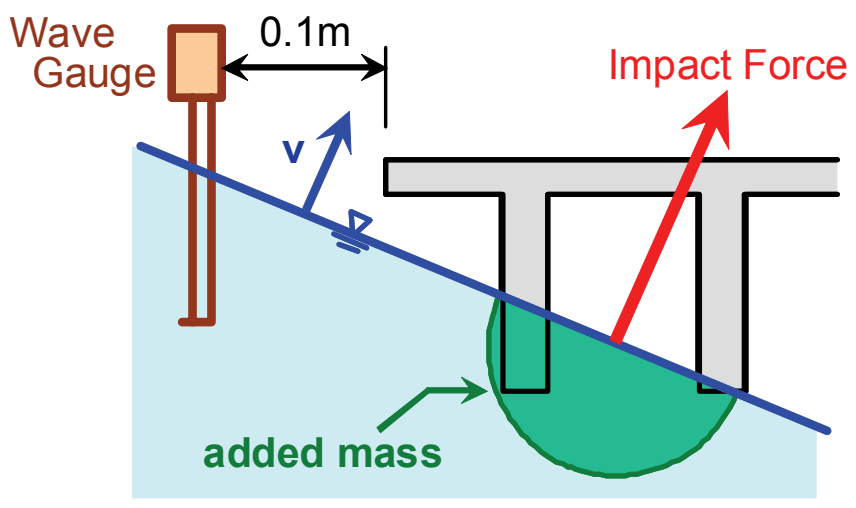

Figure 9. Added mass of model bridge. 


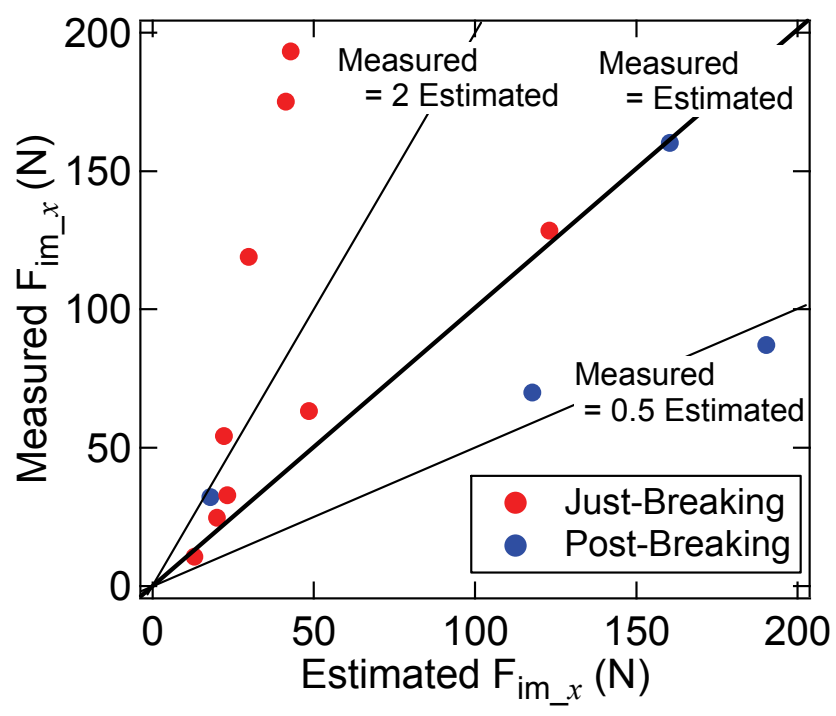

(a) Horizontal component

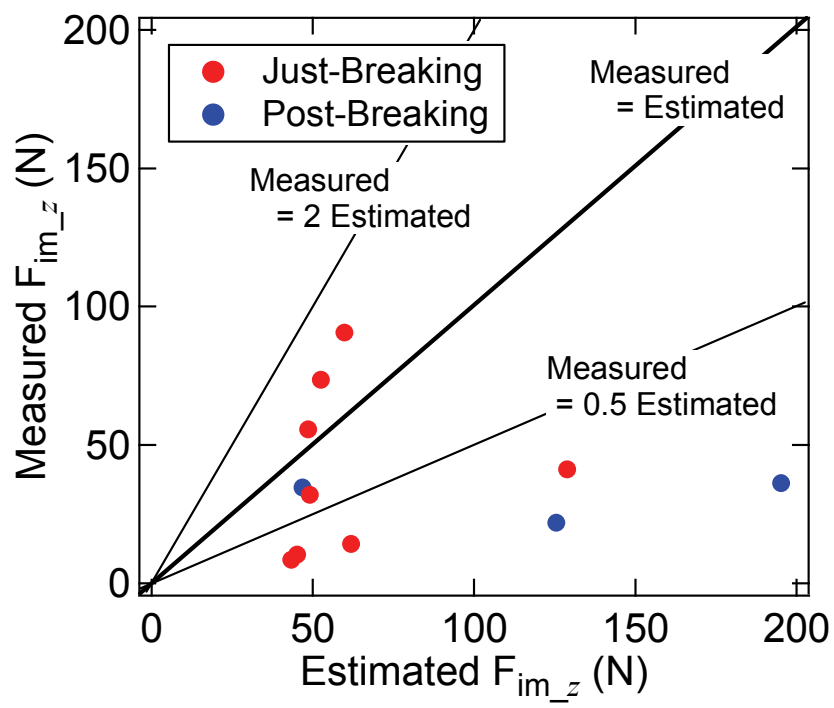

(b) Vertical component

Figure 10. Vertical components of measured and estimated impact fluid force.

\section{Characteristics of Wave Pressure}

Figure 11(a) shows the relationship between the maximum wave pressures $\mathrm{P} 1_{\max }$ and $\mathrm{P} 2_{\max }$ and the tsunami height above the model bridge. The wave pressures P1 and P2 acting on the seaward side of the main girder correspond to the horizontal fluid force. The vertical axis shows the maximum wave pressure normalized by the static pressure acting on the model bridge caused by the water level equivalent to the tsunami height above the model bridge. The horizontal axis shows the tsunami height above the model bridge normalized by the tsunami height. The larger normalized maximum wave pressure was measured in the range of the smaller normalized tsunami height above the model bridge. In the experiments conducted by Nouri et al. (2010), the wave pressure due to bore acting on the lower part of a cylindrical structure placed on a dry bed was larger than that acting on the upper part of the structure. In this experiment, the maximum wave pressure acting on the lower part of the model bridge $\mathrm{P} 1_{\max }$ was larger than that acting on the upper part of the model bridge $\mathrm{P} 2_{\max }$ in several cases, 
especially in the range of the larger normalized tsunami height above the model bridge. However, there was no clear relationship between the maximum wave pressures $\mathrm{P} 1_{\max }$ and $\mathrm{P} 2_{\max }$ because the ratio of the distance between the pressure gauges $\mathrm{P} 1$ and $\mathrm{P} 2$ to the tsunami height was small.

Figure 11(b) shows the relationship between the maximum wave pressures $\mathrm{P} 3_{\max }$ and $\mathrm{P} 4_{\max }$ and the tsunami height above the model bridge. The wave pressures P3 and P4 correspond to the uplift force. The normalized maximum wave pressure $\mathrm{P} 4_{\max }$ slightly increases with the decrease in the normalized tsunami height above the model bridge; however, the normalized maximum wave pressure $\mathrm{P} 3_{\max }$ rapidly increases with the decrease in the value of the horizontal axis. The maximum wave pressure $\mathrm{P} 3_{\max }$ was larger than the maximum wave pressure $\mathrm{P} 4_{\max }$ in almost all of the cases. From Figure 11, the normalized maximum pressures were larger in the cases where the crest of the larger incident wave hit the model bridge.

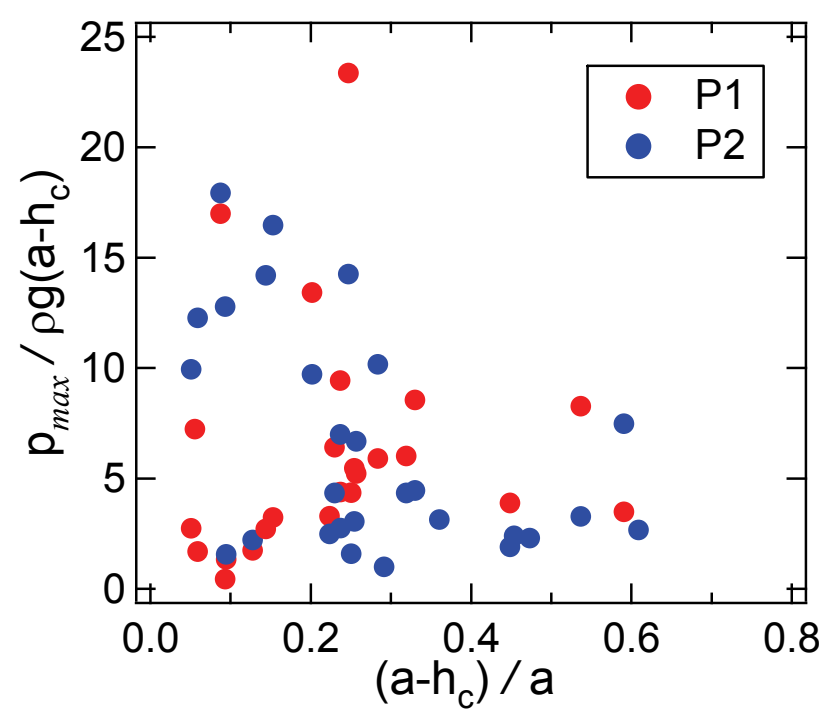

(a) P1max and P2max

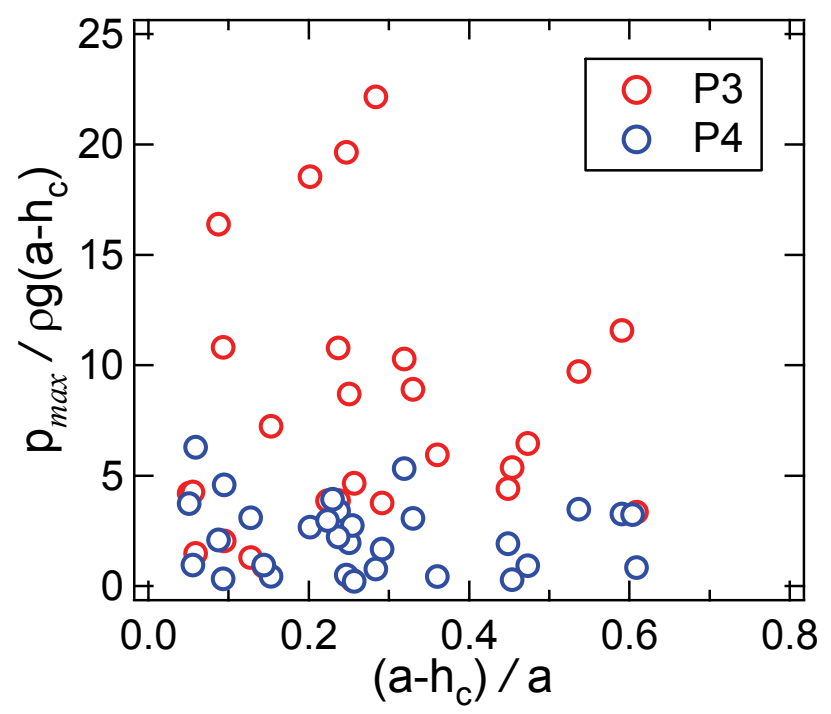

(b) P3max and P4max

Figure 11. Relationship between maximum wave pressures and tsunami height above model bridge. 


\section{Estimation of Wave Pressure}

Figure 12 shows the comparison between the measured and the predicted wave pressures acting on the seaward side of the girder of the model bridge. The wave pressure was estimated by the following equations proposed by Tanimoto (1983) and Tanimoto et al. (1983) for predicting wave pressure due to tsunamis acting on vertical walls.

$$
\begin{gathered}
p=2.2 \rho g a \\
\eta^{*}=3.0 a
\end{gathered}
$$

where $\eta^{*}$ is the elevation to which the wave pressure is exerted.

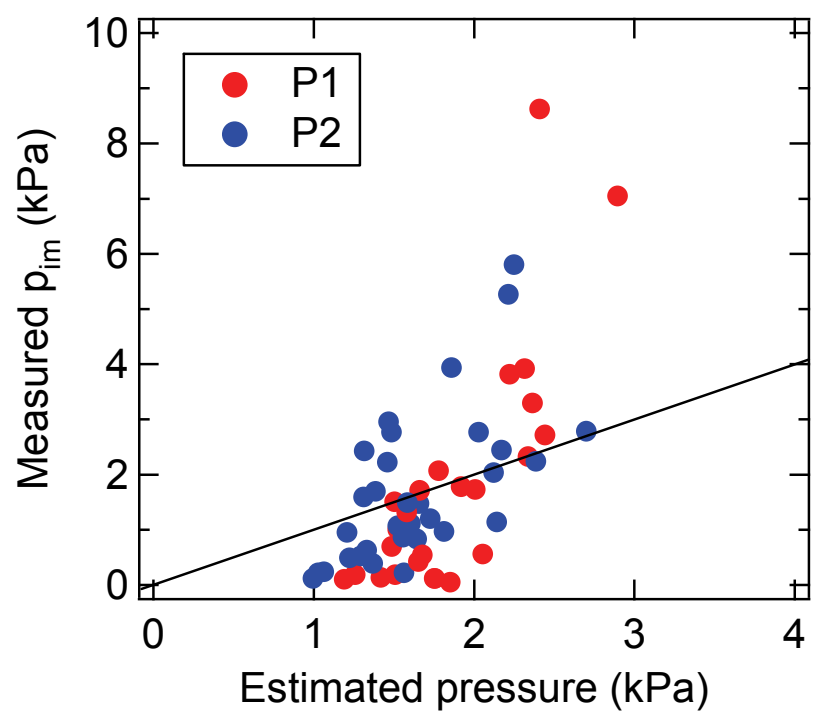

(a) Impact wave pressure

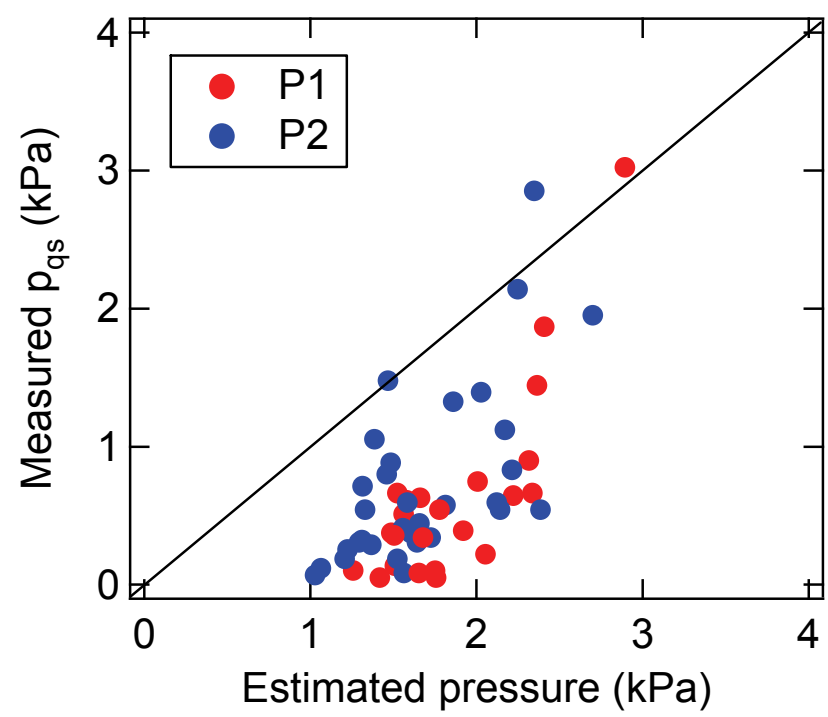

(b) Quasi-static wave pressure

Figure 12. Relationship between measured and estimated wave pressure on model bridge. 
In Japanese guidelines for coastal and harbor structures, it is mentioned that these equations can be used for estimating wave pressure due to tsunamis acting on vertical walls. Although the applicability of these equations to bridge beams which have the clearance under them has not been investigated yet, the equations were used for estimating wave pressure for comparison.

Figure 12(a) shows the comparison between the peak of the measured impact pressure $p_{i m}$ and the estimated wave pressure. In many cases, the peaks of the measured impact pressures were underestimated for both P1 and P2. Figure 12(b) shows the comparison between the measured quasistatic pressure $p_{q s}$ and the estimated pressure. In almost all of the cases, the quasi static pressure was estimated on the safe side. The method for estimating the impact pressure has to be investigated more in addition to the influence of the impact pressure on the stability of bridges.

\section{STABILITY OF BRIDGE}

The stability of the prototype girder bridge with three main girders was briefly investigated. In this study, critical force for the stability was briefly estimated on the assumption that the bridge was washed away by breakage of the bolts used in the shoes. The bolts were assumed to be broken by shear stress. The length and the width of the prototype girder bridge were assumed to be $24.0 \mathrm{~m}$ and $7.5 \mathrm{~m}$,
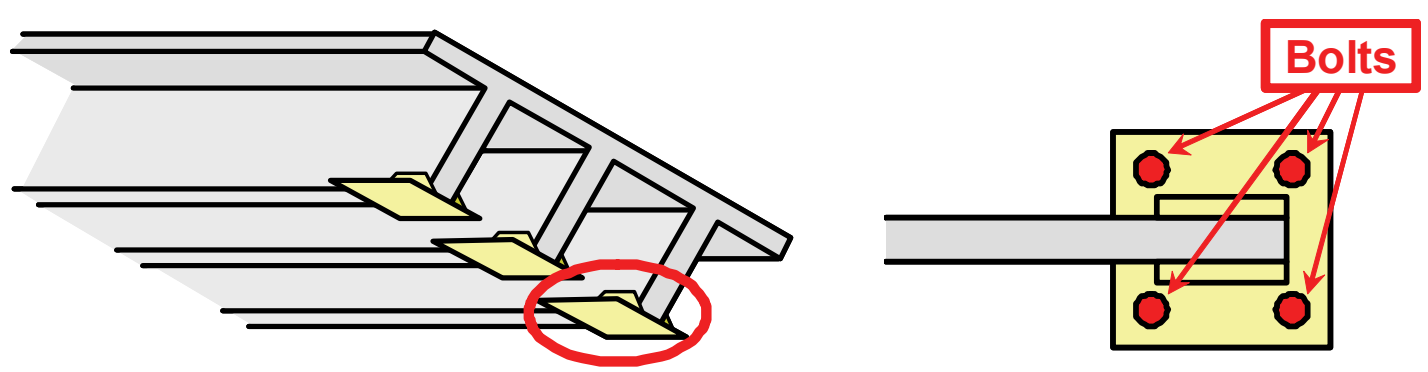

Figure 13. Schematic sketch of shoes supporting bridge.

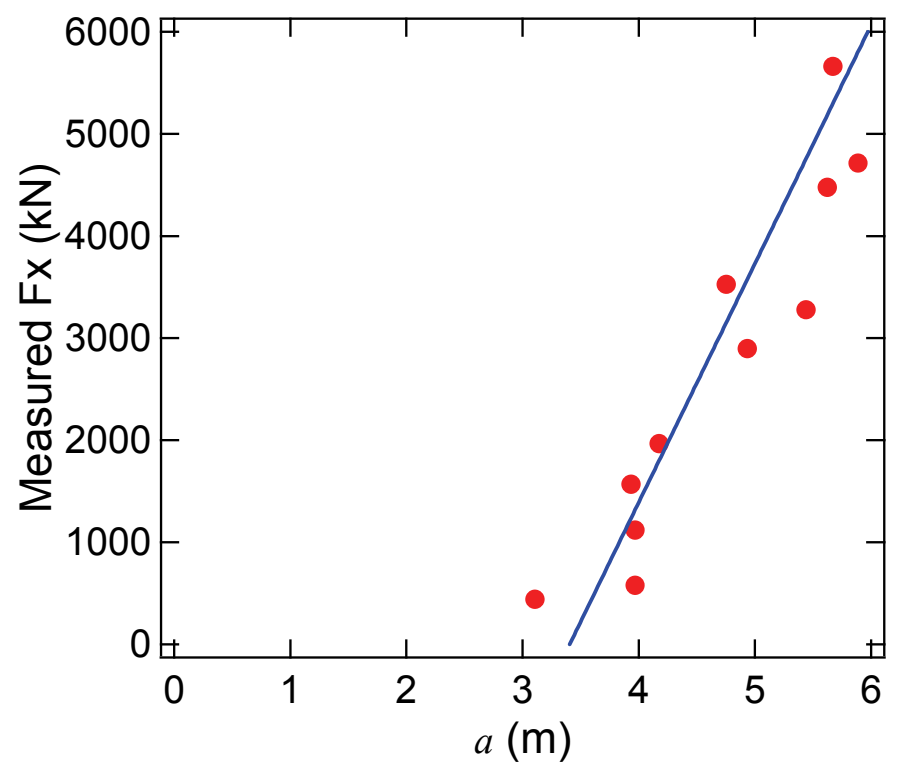

Figure 14. Relationship between horizontal fluid force and tsunami height. 
respectively. The mass of the bridge was calculated to be $160.2 \mathrm{t}$ including the mass of the slab and the pavement. The bridge was supported by three shoes on each side and each shoe was assumed to be fixed by four bolts shown in Figure 13. In other words, the bridge was assumed to be fixed by 24 bolts in total. The diameter of the bolt was assumed to be $18 \mathrm{~mm}$ and the strength of the bolt against shear was assumed to be $192 \mathrm{~N} / \mathrm{mm}^{2}$ (used steel: SD295A). Therefore, the strength of the bridge against shear was estimated to be $1171.1 \mathrm{kN}$.

Figure 14 shows the relationship between the horizontal fluid force and the tsunami height in prototype. The blue line in the figure was calculated from regression analysis. The horizontal fluid force in prototype was calculated from the horizontal fluid force $F_{x}$ measured in the case of $h=22.0$ $\mathrm{cm}$ and $h_{c}=7.5 \mathrm{~cm}$ on the basis of only the length scale of the bridge length. The measured force in prototype in the vertical axis may include the scale effect. However, the strength of the bridge against shear was compared with the measured horizontal fluid force from this figure for simplicity. In this test condition, the horizontal tsunami fluid force exceeded the strength of the bridge against shear $1171.1 \mathrm{kN}$ when the tsunami height $a$ was larger than approximately $4 \mathrm{~m}$.

\section{CONCLUSION}

In this study, the horizontal and vertical components of the tsunami fluid force and wave pressures acting on the model girder bridge were measured through the hydraulic experiments and the characteristics of the tsunami fluid force and wave pressure were investigated. The obtained results are summarized as follows:

- In the time history of the vertical component of the tsunami fluid force $F_{z}$, two peaks were measured in the case of a just breaking wave while just one peak was measured in the case of a post breaking wave.

- The horizontal and vertical components of the maximum fluid forces in the case of a post breaking wave were smaller than those in the case of a just breaking wave.

- The impact force was estimated on the basis of the change in the added mass of the model bridge in the transition from emerged to submerged conditions. The horizontal component of the impact force for just breaking waves was considerably underestimated in several cases.

- The normalized maximum wave pressure on the model bridge increased with the decrease in the normalized tsunami height above the model bridge.

- The wave pressure acting on the seaward side of the girder of the model bridge was estimated by the equation proposed by Tanimoto et al. (1983). The quasi-static pressure was estimated on the safe side in almost all of the cases while the peak of the impact pressure was underestimated in many cases.

- The stability of girder bridges was investigated using the measured tsunami fluid force. Although the scale effect on the fluid force was not taken into account in the analysis, the critical force for the stability was estimated as the strength of bolts used in the shoes against shear stress.

- In order to investigate the influence of the impact force on the stability of the bridge, the dynamic response of the bridge to the fluid force has to be analyzed because the duration of the impact force is very short.

\section{ACKNOWLEDGMENTS}

Several parts of this research were financially supported by Foundation of River and Watershed Environment Management, for which the authors expresses their gratitude.

\section{REFERENCES}

Araki, S., S. Itoh, and I. Deguchi. 2008. Experimental study on fluid force on bridge beam due to tsunami, Proceedings of the $18^{\text {th }}$ International Offshore and Polar Engineering Conference, ISOPE, Vol. III, 586-591.

Araki, S., and I. Deguchi. 2009. Experimental study on tsunami fluid force on bridge across narrow river, Proceedings of the $5^{\text {th }}$ International Conference on Asian and Pacific Coasts, Vol. 1, 143150 . 
Asakura, R., K. Iwase, T. Ikeya, M. Takao, T. Kaneko, N. Fujii and M. Omori. 2000. An experimental study on wave force acting on on-shore structures due to overflowng tsunamis, Proceedings of Coastal Engineering, JSCE, Vol. 47, 911-915 (in Japanese).

Cross, R. H. 1967. Tsunami surge forces, Journal of Waterways and Harbor Division, ASCE, Vol. 93, No. 4, 201-231.

Ikeno, M., M. Matsuyama, T. Sakakiyama, and M. Yanagisawa. 2005. Experimental study on tsunami force acting on breakwater with Soliton Fission and breaking of split waves, Annual Journal of Coastal Engineering, JSCE, Vol. 52, 751-755 (in Japanese).

Matsutomi, H. 1991. An experimental study on pressure and total force due to bore, Proceedings of Coastal Engineering, JSCE, Vol. 38, 626-630 (in Japanese).

Mizutani, N., T. Tomita, Y. Takagi, and S. Miyajima. 2006. Drag and inertia forces due to tsunami acting on a container resting on apron, Proceedings of the $30^{\text {th }}$ International Conference on Coastal Engineering, Vol. 2, 1579-1589.

Mizutani, S. and F. Imamura. 2000. Hydraulic experimental study on wave force of a bore acting on a structure, Proceedings of Coastal Engineering, JSCE, Vol. 47, 946-950 (in Japanese)

Nouri, Y., I. Nistor, and D. Palermo. 2010. Experimental investigation of tsunami impact on free standing structures, Coastal Engineering Journal, Vol. 52, No. 1, 43-70.

Ramsden, J. D., and F. Raichlen. 1990. Forces on vertical wall caused by incident bores, Journal of waterway, Port, Coastal, and Ocean Engineering, ASCE, Vol. 116, No. 5, 592-613.

Shoji, G., and Y. Mori. 2006. Hydraulic model experiment to simulate the damage of a bridge deck subjected to tsunamis, Annual Journal of Coastal Engineering, JSCE, Vol. 53, pp. 801-805 (in Japanese)

Tanimoto, K. 1983. On the hydraulic aspects of tsunami breakwaters in Japan, Proceedings of the International Tsunami Symposium 1981, IUGG, Tsunami Commission, 423-435

Tanimoto, K., T. Takayama, K. Murakami, S. Murata, H. Tsuruya, S. Takahashi, M. Morikawa, Y. Yoshimoto, S. Nakano, and T. Hiraishi. 1983. Field and laboratory investigations of the tsunami caused by 1983 Nihonkai Chubu Earthquake, Technical Note of the Port and Harbor Research Institute, No. 470, 1-299 (in Japanese). 